\title{
Surgical Options in Management of the Breast and Axilla: Independent Choices?
}

\author{
Jeffrey E. Johnson, MD¹, David W. Ollila, MD', and Judy C. Boughey, MD ${ }^{2}$ \\ ${ }^{1}$ Division of Surgical Oncology, UNC School of Medicine, Chapel Hill, NC; ${ }^{2}$ Department of Surgery, Mayo Clinic, \\ Rochester, MN
}

De-escalating the extent of axillary surgery and thus diminishing the associated morbidity is a focus of breast surgery. Although the theoretical basis for narrowing the indications for the Halstedian approach to radical lymphadenectomy has permeated surgical oncology for decades - that lymph node metastases are "indicators, but not governors, of survival" - it was the landmark publication by Giuliano et al. of ACOSOG Z0011 that solidified that concept of de-escalating axillary management in earlystage breast cancer patients treated with breast-conserving therapy (BCT). ${ }^{1-3}$ However, the conclusions and application of Z0011 in practice are not without controversy and the boundaries defining who would benefit from completion axillary lymph node dissection (cALND) or axillary radiation are not yet clearly defined.

Vane et al. demonstrate that surgical management of the axilla in women with one to two positive sentinel lymph nodes (SLNs) depends in part on the surgical management of the breast, i.e., whether patients and providers decide to pursue BCT or mastectomy. ${ }^{4}$ In a single-institution, prospectively maintained database over a 6 -year period, among 306 patients with cT1-2, cN0 disease who were found to have 1-2 positive SLNs, 199 (65\%) underwent BCT and 107 (35\%) underwent mastectomy, with 27\% and $71 \%$ respectively undergoing cALND. The authors present this as an opportunity: If axillary management is to be

(C) Society of Surgical Oncology 2021

First Received: 25 January 2021

Accepted: 26 January 2021;

Published Online: 11 February 2021

J. C. Boughey, MD

e-mail: boughey.judy@mayo.edu guided by Z0011, how many patients who elect mastectomy undergo cALND that could be avoided by choosing $\mathrm{BCT}$ ?

The question is an important one and warrants further exploration. As the treatment algorithm in this study is predicated on the results of Z0011, generalizability must be considered given differences in the clinicopathologic characteristics of this cohort (e.g., age, tumor size, hormone receptor status), extent of axillary disease (presence of macrometastases and nonsentinel node metastases), and patient selection (women and providers choosing mastectomy). The higher nodal burden in the present study is especially striking, with $75 \%$ of patients with macrometastases compared with 59\% in Z0011 and 52\% of patients with nonsentinel node metastases compared with $27 \%$ in Z0011. In practice, providers at this single institution were not strictly constrained by Z0011 criteria: $29 \%$ of patients with positive nodes after mastectomy did not undergo cALND, and $12 \%$ of BCT patients without extracapsular extension underwent cALND. Thus, the authors show that, in their institution, patient selection is critical when questions of axillary management are occurring in a more complex, higher-risk cohort than that enrolled in the Z0011 clinical trial.

It is difficult to retrospectively apply a treatment algorithm to select patients when all of the information that contributed to a clinical decision is not subsequently available for analysis. In this cohort of cT1-2, cN0 disease, where $65 \%$ of women chose BCT and $35 \%$ mastectomy, the authors argue that more women would have conserved their axilla had they chosen BCT. They note that some patients would be less likely to choose BCT, such as those with deleterious BRCA mutation or multifocal or multicentric disease. For context, evaluating a cohort from Mayo Clinic Rochester of 1723 patients with cT1-2, cN0 disease 
treated with mastectomy, only 963 (55.9\%) had unifocal tumor with size $<4 \mathrm{~cm}$, while 691 had multifocal or multicentric disease and 69 had tumor $\geq 4 \mathrm{~cm}$ and thus may not have been appropriate candidates for BCT, depending on breast size and pattern of disease (Boughey JC, unpublished data, January 2021). Furthermore, no data are provided by the authors on other clinical factors that may push a patient toward mastectomy, the relative clinical contraindications to adjuvant radiotherapy, or other incommensurable factors that women and providers weigh when choosing a treatment course. ${ }^{5}$ Overall, it is unclear whether the contingent increased risk of undergoing cALND after electing mastectomy would tip the scales toward BCT for many women. Without a full accounting of why women chose mastectomy, it is difficult to argue that all or even most women in this study could or would have pursued BCT.

The authors note that as a way of helping women avoid axillary dissection, the risk of cALND should be discussed with patients and should weigh in the guidance provided in the shared decision-making process between BCT and mastectomy. However, this is difficult to conceptualize and implement in a busy clinical breast practice. Who should be counseled, what risks should be discussed, how best to do this without overwhelming the patient, and how many patients would ultimately decide against mastectomy to avoid a potential need for cALND? The authors recommend counseling only those patients who choose mastectomy about the "additional" risk of ALND and axillary morbidity. Whereas Vane et al. reported on patients with cT1-2, cN0 disease found to have one to two positive SLNs, this is only a small subgroup of all women in their practice with $\mathrm{cT} 1-2, \mathrm{cN} 0$ disease who are seen and counselled regarding surgical options; the vast majority of patients undergoing mastectomy would not have a change in axillary management even if they were able to elect BCT as the vast majority are node negative (approximately $>75 \%$ ) and some of those that are node-positive have more than two positive SLNs. Furthermore, the "additional" risk related to the axilla secondary to mastectomy cannot be defined by the data here and would better be measured with summative outcomes data. It is likely that the number need to be counseled in order to avoid an unnecessary axillary complication is quite high, and, without clearly quantified risks, there is the likelihood for most patients of complicating an already involved discussion with marginal benefit.

The decision to avoid cALND is not isolated from the interacting network of risks otherwise associated with $\mathrm{BCT}$, mastectomy, radiation, reconstruction, etc. Because it is difficult to identify the risks of electing mastectomy a priori, it would be more valuable to focus on avoiding potential overtreatment of the axilla in those patients who undergo mastectomy and are found to have one to two positive SLNs. For patients with positive SLNs options for axillary management after mastectomy include cALND, axillary radiation, neither or both. Thoughtful, multidisciplinary, team discussion can help minimize overtreatment in the setting of one to two positive nodes. At Mayo Clinic, our multidisciplinary guidelines recommend omission of both ALND and axillary radiation in patients with low-risk criteria defined as pT1-T2 tumor $(<5 \mathrm{~cm}$ in size $)$ with isolated tumor cells or micrometastatic foci $(\leq 2 \mathrm{~mm})$ in a single sentinel node. For patients at intermediate risk, we recommend either axillary radiation if PMRT is otherwise indicated or CALND if no PMRT is planned. This leaves combined cALND and PMRT for patients at the highest risk, such as those with three or more positive SLNs, gross extranodal extension, or other risk factors for recurrence. Similar guidelines are recommended at other sites. ${ }^{6}$

Ultimately, the critical question is: How should the axilla be managed in early-stage breast cancer patients undergoing mastectomy? We have several years to wait for the completion of several, randomized, controlled clinical trials that address this question, as listed in Table 1. While the trials are all intended to expand on Z0011 and include patients treated with both BCT and mastectomy, they have slightly different inclusion/exclusion criteria, interventions, primary outcomes, adjuvant radiation protocols, and use of ultrasound to screen the axilla. ${ }^{7-11}$ The diversity of the trials offers a more comprehensive path forward at the risk of obfuscation in the case of contradictory results. Regardless, these trials should offer level I evidence on the omission of cALND after mastectomy and may offer secondary results on clinical outcomes between BCT and mastectomy.

Overall, Vane et al. demonstrate that in current clinical practice, surgical axillary management depends in part on the breast surgical procedure and present this as an opportunity to further refine clinical practice. It is important that the breast surgeon understands that the decision for a mastectomy potentially influences axillary management in patients found to be sentinel node positive and where appropriate discuss the implications with patients. Where management algorithms depart from existing level I clinical trial data, there is a critical role for multidisciplinary team collaboration, consideration of alternative treatment algorithms, and thoughtful shared decisionmaking with patients. Ultimately, we look forward in the coming years to data from the ongoing trials that may help to resolve these questions, just as Giuliano et al. did with ACOSOG Z0011 for the prior era. 


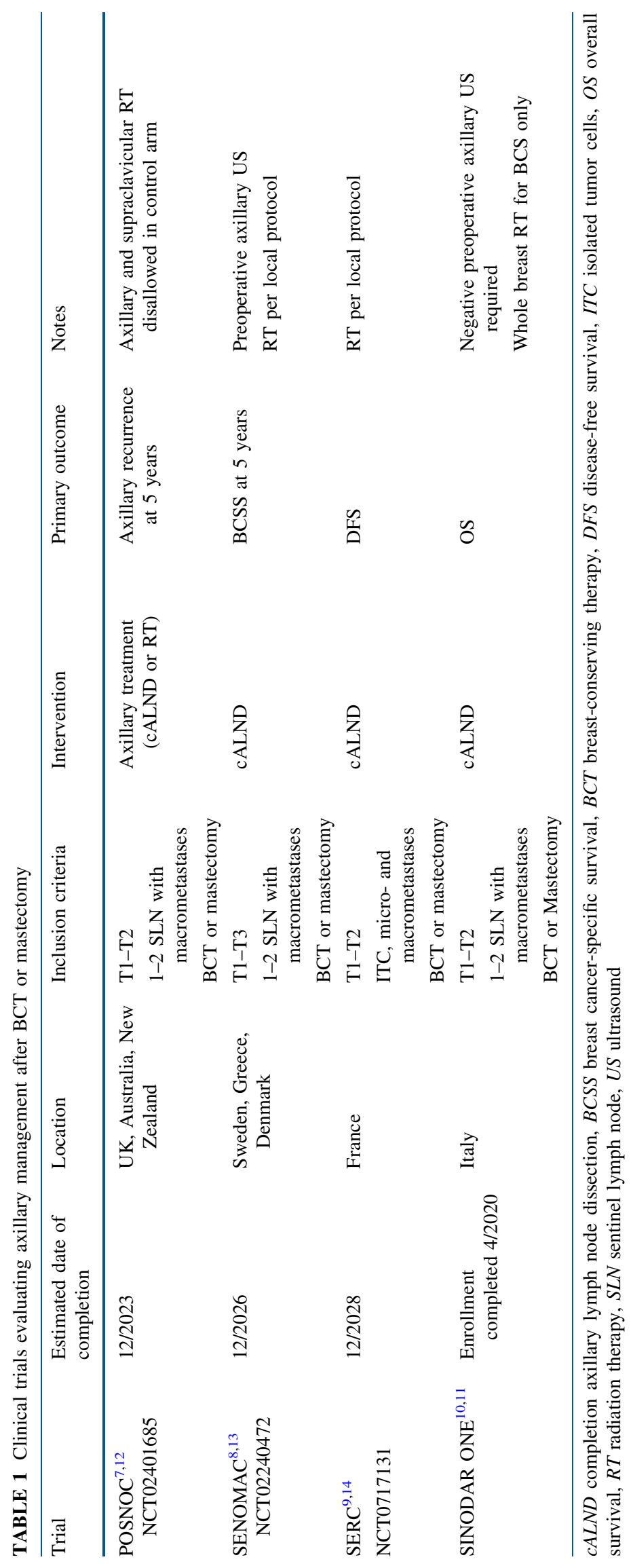


DISCLOSURES The authors declare no conflict of interest.

\section{REFERENCES}

1. Cady B. Basic principles in surgical oncology. Arch Surg. 1997;132(4):338-46.

2. Giuliano AE, Hunt KK, Ballman KV, et al. Axillary dissection vs no axillary dissection in women with invasive breast cancer and sentinel node metastasis: a randomized clinical trial. JAMA. 2011;305(6):569-75.

3. Giuliano AE, Ballman KV, McCall L, et al. Effect of axillary dissection vs no axillary dissection on 10-year overall survival among women with invasive breast cancer and sentinel node metastasis: the ACOSOG Z0011 (Alliance) randomized clinical trial. JAMA. 2017;318(10):918-26.

4. Vane MLG, Hunter-Squires J, Kim S, Smidt ML, Giuliano AE. Women could avoid axillary lymph node dissection by choosing breast-conserving therapy instead of mastectomy. Ann Surg Oncol. 2021. https://doi.org/10.1245/s10434-021-09674-9.

5. Gu J, Groot G, Boden C, Busch A, Holtslander L, Lim H. Review of factors influencing women's choice of mastectomy versus breast conserving therapy in early stage breast cancer: a systematic review. Clin Breast Cancer. 2018;18(4):e539-54.

6. Morrow M. Management of the node-positive axilla in breast cancer in 2017: selecting the right option. JAMA Oncol. 2018;4(2):250-1.

7. Goyal A, Dodwell D. POSNOC: a randomised trial looking at axillary treatment in women with one or two sentinel nodes with macrometastases. Clin Oncol $(R$ Coll Radiol $)$. 2015;27(12):692-5.

8. de Boniface J, Frisell J, Andersson Y, et al. Survival and axillary recurrence following sentinel node-positive breast cancer without completion axillary lymph node dissection: the randomized controlled SENOMAC Trial. BMC Cancer. 2017;17(1):379.
9. Houvenaeghel G, Cohen M, Raro P, et al. Overview of the pathological results and treatment characteristics in the first 1000 patients randomized in the serc trial: axillary dissection versus no axillary dissection in patients with involved sentinel node. BMC Cancer. 2018;18(1):1153.

10. Tinterri C, Canavese G, Bruzzi P, Dozin B. SINODAR ONE, an ongoing randomized clinical trial to assess the role of axillary surgery in breast cancer patients with one or two macrometastatic sentinel nodes. Breast. 2016;30:197-200.

11. Tinterri C, Marrazzo E, Anghelone C, et al. Abstract PD4-01: preservation of axillary lymph nodes compared to complete dissection in T1-T2 breast cancer patients presenting 1-2 metastatic sentinel lymph nodes: a multicenter randomized clinical trial Sinodar One. In: Proceedings of the 2019 San Antonio Breast Cancer Symposium. December 8-12, 2020.

12. Goyal A. POSNOC - a trial looking at axillary treatment in early breast cancer. ClinicalTrials.gov. Available at: https://clinicaltria 1s.gov/ct2/show/NCT02401685. Updated April 6, 2020. Accessed 16 Jan 2021.

13. de Boniface J. Sentinel node biopsy in breast cancer: omission of axillary clearance after macrometastases. A randomized trial. ClinicalTrials.gov. Available at: https://clinicaltrials.gov/ct2/sho w/NCT02240472. Updated September 9, 2020. Accessed 16 Jan 2021

14. Houvenaeghel G. Axillary node dissection versus no dissection in breast cancer with positive sentinel lymph node. Available at: $h$ ttps://clinicaltrials.gov/ct2/show/NCT01717131. Updated January 22, 2020. Accessed 16 Jan 2021.

Publisher's Note Springer Nature remains neutral with regard to jurisdictional claims in published maps and institutional affiliations. 